\title{
The Sorbent Mass Variation Method: a New Possibility for Determination of Binding
}

\section{Isotherms}

\author{
Miklós Nagy*, Zoltán Siegl, Krisztina Szili, Viktória Fábos and Krisztina Kántor
}

Institute of Chemistry, Department of Physical Chemistry, Laboratory for Colloid and Supermolecular Structures, L. Eötvös University, P.O. Box 32 H-1518 Budapest 112, Hungary

\begin{abstract}
Measurement of equilibrium mass fraction of a surfactant as a function of the sorbent mass fraction was performed on gel sorbent-solution systems in order to determine binding isotherms and to calculate fundamental characteristics of the solvation layer. With application of this new method it was possible to calculate specific solvation/sorption capacity and absolute average local composition of the solvation layer. It has been pointed out by systematic variation of the composition (hydrophobicity) and degree of cross-linking of the gel sorbents that the ratio of components in the solvation layer can be constant in a given range of equilibrium mass fraction of the sodium dodecylsulfate (SDS) and that the specific solvation/sorption capacity of gel sorbents can be much greater than that of activated carbon type adsorbents. On the basis of a mixed sorbent models it turned out from calculations that there is no preferential binding of SDS close to the chemical cross-links and the surfactant molecules prefer vinyl acetate groups as binding sites. The density of cross-links regulates the aggregation number of the bound surfactant as well. For loose gels both binding isotherms and swelling curves show that the surfactant-polymer interaction is a strongly cooperative process. The result of these experiments may influence general concept of solvation/sorption isotherms and all related phenomena.
\end{abstract}




\section{INTRODUCTION}

The concept of phase introduced by J. Willard Gibbs defines perhaps the most important phenomenological model which is applied successfully in many branches of chemical science. Without this, for instance, the thermodynamics or kinetics of chemical reactions could not have been developed. Definition of concentration of a given component in a system assumes also homogeneous spatial distribution of all components. However, crossing the boundary of contacting phases, changes in many properties take place localized at the interface due to the asymmetry of intermolecular forces at the interfacial zone. Not only a mechanical effect (interfacial tension) appears, but considerable difference, positive or negative, can occur between the local and bulk concentration. The local enrichment of one of the components of a mixture or solution may be called adsorption. The widespread occurrence of this interfacial phenomenon in the natural and artificial world as well as its many practical applications have been maintaining interest toward this field of colloid and interface science for long time. ${ }^{1-8}$ However, such selective binding of one of the components of a mixture by a third component (sorbent) may frequently occur not only at different types of interfaces but inside homogeneous molecular systems like in electrolyte $e^{9-13}$, nonelectrolyte ${ }^{14,15}$ and polymer ${ }^{16-20}$ solutions, too. This latter phenomenon is called selective or preferential solvation. It can be responsible for some kinetic 'solvent' effect and it may influence determination of molar mass of macromolecules in mixed solvents by light scattering (LS). ${ }^{19}$ It can play important role in the conformational changes of biopolymers, too. ${ }^{21}$

The experimental characterization of adsorption and preferential solvation depends on the separability of the sorbent together with its microenvironment from the rest of bulk solution. So, for instance, in the case of solid/liquid (S/L) interface the solid sorbent is usually in a separate 
phase hence it is possible to determine the change in concentration (mole fraction) of the equilibrium (supernatant) solution phase with the application of a suitable analytical procedure. Separation is also possible for polymer solutions by making use of equilibrium dialysis. ${ }^{22,23}$ Amongst the methods without separation different kinds of spectroscopy (i.e. UV, Vis, NMR) and light scattering need to be mentioned. ${ }^{16,24,25,26}$ Such in situ measurements, however, often require additional assumptions in order to be able to provide information on local concentration. $^{27}$

In order to obtain an adsorption isotherm, in overwhelming majority of cases, the so-called 'batch' method is applied. In this case the equilibrium concentration (mole fraction) of one of the component of the mixture is measured at fixed value of $m_{\mathrm{t}} / m_{\mathrm{s}}$ but at different initial mole fractions. Here $m_{\mathrm{t}}$ is the mass of mixture or solution, $m_{\mathrm{s}}$ is mass of sorbent and the ratio $m_{\mathrm{t}} / m_{\mathrm{s}}$ is usually less than 10. It is a generally accepted view that the shape of isotherm does not depend on the ratio $m_{\mathrm{t}} / m_{\mathrm{s}}$; they are solely a function of the equilibrium mole fraction. ${ }^{28}$

In one of our earlier study ${ }^{29}$ adsorption experiments have been carried out in a radically different manner from the rest of literature: at constant mass of the mixture and initial mass fraction of one of the mixture component, $w_{1, i}$, the mass of sorbent was varied. In other words: at fixed $m_{\mathrm{t}}$ and $w_{1, \mathrm{i}}$ the mass of adsorbent or $w_{\mathrm{s}}$, the mass fraction of the adsorbent in the adsorption system was used as an independent variable. This treatment now may be called Sorbent Mass Variation (SMV) method. It has been shown by adsorption experiments performed on activated carbon-dilute aqueous 1-propanol system that the equilibrium mass fraction, $w_{1, \mathrm{e}}$, of 1-propanol was an unambiguous function of the sorbent mass fraction in a rather wide range $\left(3<m_{\mathrm{t}} / m_{\mathrm{s}}<100\right)$. The positions of the $w_{1, \mathrm{e}}=\mathrm{f}\left(w_{\mathrm{s}}\right)$ functions were determined only by the initial mass fraction, $w_{1, \mathrm{i}}$. From this group of functions, at strictly constant $w_{\mathrm{s}}$ 
values, the $m_{1}^{\sigma}=f\left(w_{1, e}\right)$ function, i.e. the adsorption isotherm could have been calculated. Here $m_{1}^{\sigma}$ is the excess amount adsorbed expressed by masses. To our greatest surprise, instead of a single curve, a group of functions were obtained. Namely, some parts of the SMV type of isotherms have separated from the so-called experimental upper limiting function showing a flat maximum and a linear or nearly linear descending part. Assuming constant specific capacity and layer composition along linear parts, both specific adsorption capacity and local mass fraction of 1-propanol could have been calculated for each SMV isotherm. (See below Theory section for further detailes.) To the best of our knowledge this was the first time when fundamental adsorption data were obtained from measurements performed only at a fixed $w_{1, \mathrm{i}}$.

This paper is a continuation of our previous systematic studies on polymer gels and adsorption. Swollen synthetic polymer networks as sorbents have been chosen because inside they have no definite surface, the binding (preferential solvation) occurs in a separable, homogeneous phase and their chemical composition and structure can be much better varied and characterized than those of adsorbents based on activated carbon. It is known from the literature $^{30}$ that surfactants have binding isotherms of peculiar shape; therefore they can serve as good models for such investigations. Since SDS is a frequently used model in physico and colloid chemical studies and it has many practical applications, we believe that the complex approach and the SMV method together will results in a new and deeper insight into the nature of these very complicated polymer gel-surfactant systems.

In the present work first the condition of applicability of the adsorption formalism to swollen gels will be analysed and then a systematic experimental investigation of binding of SDS into 
poly(vinyl alcohol) based hydrogels of various compositions (hydrophobicity) and degree of chemical cross-linking will be presented and discussed.

\section{THEORY}

General Characterization of Adsorption Excesses. For quantitative characterization of adsorption/solvation layer the knowledge of local ratio of the components, the extent and structure of the adsorption/solvation layer would be necessary as a function of the composition of bulk solution. In majority of cases, however, only a quantity the so-called adsorption excess is available from experimental data. For instance, at an S/L type of interface, according to the widely used definition (recommended also by IUPAC) ${ }^{31}$

$$
n_{i}^{\sigma(n)}=n^{0} x_{i}^{0}-n^{0} x_{i}^{l}=n^{0} \Delta x_{i}^{l}
$$

where $n_{i}^{\sigma(n)}$ is the surface excess amount of component $\mathrm{i}, n^{0}$ is the total amount of mixture with an initial mole fraction $x_{i}^{0}$, which, when equilibrated with a sorbent of mass $m_{s}$, changes to $x_{i}^{l}$. A specific surface excess, $n^{0} \Delta x_{i}^{l} / m_{s}$ can also be defined, or the surface excess amount can be referred to unit surface area of the adsorbent (surface excess concentration, $\Gamma_{\mathrm{i}}^{(\mathrm{n})}$ ). Eq 1 essentially a measuring instruction and in itself it does not contain information on properties of the adsorption layer. There can be given, however, another relationship ${ }^{28}$

$$
n_{i}^{\sigma}=n^{0} \Delta x_{i}^{l} / m=n_{i}^{s}-n^{s} x_{i}^{l}=n^{s}\left(x_{i}^{s}-x_{i}^{l}\right),
$$

where $n_{i}^{\sigma}$ is the specific surface excess, $n_{i}^{s}$ is the amount of component $i$ at the surface and $n^{s}=\sum_{i} n_{i}^{s}$ is the total amount of the components in the adsorption layer referred to unit mass of adsorbent (specific adsorption capacity); $x_{i}^{s}$ is the mole fraction of component $\mathrm{i}$ in the 
adsorption layer. The adsorption isotherm can now be defined as a function, $n_{i}^{\sigma}=f\left(x_{i}^{l}\right)$ at constant pressure and temperature. There are many other possibilities to characterize adsorption interaction ${ }^{6,7}$ using different scales of composition. Also, instead of the $n_{i}^{\sigma}=f\left(x_{i}^{l}\right)$ function there are different kinds of representations of adsorption data which in some cases can provide more information on the adsorption layer. ${ }^{32-34}$ Thus, for some selected types of isotherms the composition of the adsorbed layer, the specific adsorption capacity and even the specific surface area of the solid adsorbent could have been approximated from adsorption data. ${ }^{7,35}$

In one of our earlier studies ${ }^{29}$ general relationships have been derived with the application of the mass balance (Law of Conservation of Mass) to a closed adsorption system. Accordingly, by making use of the original notations, ${ }^{29}$ the specific excess amount of component $1, m_{1}^{\sigma}$ is

$$
m_{1}^{\sigma}=-\left(w_{1, e}-w_{1, i}\right) \frac{m_{t}}{m_{s}}=-\left(w_{1, e}-w_{1, i}\right)\left(\frac{1}{w_{s}}-1\right)=k\left(w_{1, l}-w_{1, e}\right)
$$

Here the negative sign originates from the accepted convention $(\Delta=$ final state - initial state). $w_{1, l}$ is local (layer) average mass fraction of component 1 and $k$ is the specific adsorption capacity; $k=m_{l} / m_{\mathrm{s}}$, where $m_{l}=m_{1, l}+m_{2, l}+\ldots$ is the sum of the masses of the components in the adsorption layer. The right-hand side of eq 3 , in complete agreement with eq 2 , gives the excess in a most instructive form: it is a product of specific adsorption capacity and the mass fraction (mole fraction) difference characterizing the selectivity of an adsorbent.

Relationships for Swollen Gels. Comparison reveals that one of the important differences between an S/L system and a gel that inside gel the immobilized mixture or solution can be split into at least two parts, namely to a solvation layer and the so-called intermediate liquid, or 
recalling its old name, intermicellar liquid. In the case of swollen gels, amongst the compositional variables, degree of swelling, here the mass degree of swelling, $q_{\mathrm{m}}$, is essential which is defined as follows

$$
q_{m}=\frac{m_{s}+m_{l}+m_{\mathrm{int}}}{m_{s}}=\frac{1}{w_{s, g}}
$$

where $m_{\mathrm{s}}$ is the mass of polymer (sorbent) in the gel (and in the complete system), $w_{\mathrm{s}, \mathrm{g}}$ is the mass fraction of polymer in the swollen gel, $m_{\mathrm{int}}$ is the mass of intermicellar liquid. The relationship between the specific solvation capacity, $k^{\mathrm{sv}}$, and $q_{\mathrm{m}}$ is

$$
q_{m}=\frac{m_{s}+m_{l}+m_{\mathrm{int}}}{m_{s}}=1+k^{s v}+\frac{m_{\mathrm{int}}}{m_{s}}
$$

If $m_{\mathrm{int}}=0$, i.e. no intermicellar liquid is present in the system, then $q_{\mathrm{m}}=1+k^{s v}$. The mass balance for component 1 may be given as

$$
m_{t} w_{1, i}=m_{e} w_{1, e}+m_{\mathrm{int}} w_{1, \mathrm{int}}+m_{l} w_{1, l}
$$

From eq 6 the excess can be derived with similar algebra as has been given earlier ${ }^{29}$ :

$$
\left(m_{1}^{s v}\right)_{a p p}=-\frac{\left(w_{1, e}-w_{1, i}\right) m_{t}}{m_{s}}=k^{s v}\left(w_{1, l}-w_{1, e}\right)+\left(q_{m}-1-k^{s v}\right)\left(w_{1, \mathrm{int}}-w_{1, e}\right) .
$$

As it is seen, the right-hand side of eq 7 contains an extra term compared it with eq 3 so, it cannot likely be used directly for determination of local (layer) composition and specific solvation capacity in a way as it was previously outlined. In this case only an apparent value of the specific solvation excess can be obtained. If, however, the difference $\left(w_{1, \text { int }}-w_{1, \mathrm{e}}\right)$ and $k^{\mathrm{sv}}$ are available from experimental data a correction can be made. Fortunately, for many systems 
$w_{1, \mathrm{e}} \equiv w_{1, \text { int }}$, thus the second term becomes zero. Also, if the experimental $m_{1}^{s v}=\mathrm{f}\left(w_{1, \mathrm{e}}\right)$ function gives the expected linear portion, this is a kind of indication that the last term in the right-hand side of eq 7 is negligible. If the maximum mass degree of swelling, $q_{\mathrm{m} \text {,max }}$, is known for a system then the upper limit of $w_{\mathrm{s}}$ that can be experimentally applied is $w_{\mathrm{s}, \max } \leq 1 / q_{\mathrm{m}, \max }$. Similarly, in the case of an S/L type of interface the condition is $w_{\mathrm{s}, \max } \leq 1 / 1+k$. In both cases at larger $w_{\mathrm{s}}$ values the supernatant mixture or solution will not be present in the adsorption/solvation system.

\section{EXPERIMENTAL SECTION}

Materials. Some of the chemicals such as benzene, ethanol, $\mathrm{HCl}, \mathrm{NaOH}$, acetic acid, acetone were of analytical grade ( $\geq 99 \%$, Reanal, Hungary) and were used without purification. The sodium dodecylsulfate was purchased from Merck (for biochemistry and surfactant test, $\geq 99$ $\%$ ) and before use it was recrystallized from hot 1:1 (by volume) mixture of benzene and ethanol. The critical concentration of micelle formation (c.m.c.) was determined by electric conductivity measurements (by a conductivity meter OK-102/1, Radelkis, Hungary) and was found to be $8.2 \mathrm{mM}$. The cross-linking agent, glutaric dialdehyde (GDA), was $26.0 \mathrm{~m} / \mathrm{m}$ per cent aqueous solution (Merck) which was used after the necessary dilution. For preparation of aqueous solutions of SDS and for final purification of the swollen hydrogels double distilled water was applied.

The pure poly(vinyl alcohol) (PVA) and the random copolymers were prepared from the completely hydrolysed and partially fractionated POVAL 420 (Kuraray Co., Japan) sample. The copolymers were synthesized by reacetylating the completely hydrolysed and fractionated PVA with acetic acid in aqueous solution at $333 \mathrm{~K}$. The so-called blocky type of copolymer was 
prepared by saponification of poly(vinyl acetate) (PVAc) (Mowilith-50, Hoechst, Germany) dissolved in acetone with $\mathrm{NaOH}$. All other details are given in earlier works. ${ }^{36-38}$ Some important characteristics of the linear polymer samples are summarized in Table 1(S1, Supporting Info.). The higher softening temperature of the 'blocky' copolymer compared with that of the statistical copolymer sample indicates the presence of pure VOH blocks along the polymer chains. $^{36,37}$

Preparation of Gels. ${ }^{38}$ The hydrogels were synthesized in the form of $1 \mathrm{~mm}$ thick gel membranes in between glass sheets. The concentration of the PVA and copolymers during cross-linking reaction was 0.205 mole repeat units $/ 100 \mathrm{~cm}^{3}$ (approx. ten mass per cent). The degree of cross-linking was varied with variation of the ratio mole repeat unit/mole GDA (RU/GDA) in the range from 10 to 100 . It took nearly five days to complete the cross-linking reaction at $\mathrm{pH} 2.2-2.5$ and at $298 \mathrm{~K}$. It is important to note that the copolymers of the highest VAc content were cross-linked in 1-propanol-water mixture $(25 \mathrm{~m} / \mathrm{m}$ per cent 1-propanol) in order to keep at minimum the effect of preexisting order of the polymer solutions on structure of the final network. Then, the gel sheets were neutralized and washed exhaustively until reaching the electric conductivity of the double distilled water (specific conductivity: $\approx 1 \times 10^{-4}$ $\left.\mathrm{S} \cdot \mathrm{m}^{-1}\right)$. The purity of the washing liquid was also checked by interferometric measurements. The gel membranes were cut into smaller $(\approx 1 \times 3 \times 6 \mathrm{~mm})$ pieces which were first airdried, then dried in a vacuum oven at $333 \mathrm{~K}$ until constant mass. The dry networks were stored in a closed vessel until use. (For further detailes see, Table 2., Supporting Info. S2.)

Measurement of Sorption and Swelling. Sorption and swelling measurements were carried out at two solution masses, $m_{\mathrm{t}}$, namely at 10.0 and $30.0 \mathrm{~g}$. In the first case (10.0 g) small (12 
$\mathrm{cm}^{3}$ ) glass bottles provided with plastic cups and sealed with Parafilm, while for the larger $m_{\mathrm{t}}$ (30.0 g) plastic bottles of $34 \mathrm{~cm}^{3}$ volume sealed with airtight screwed-on cups were used. In both cases preliminary tests have shown that during measurements no evaporation loss and no observable adsorption at walls of the vessels had occurred. The time necessary to attain sorption and swelling equilibrium was found by independent measurements to be $48 \mathrm{~h}$, but during the actual experiments 3-4 days were applied. For measurements of all masses always an analytical balance (Mettler Toledo) with reproducibility $\pm 0.0001 \mathrm{~g}$ was used. The carefully dried gel sorbents with accurately known masses were trasferred in the sorption vessels (in the range $\approx$ $0.03-1.0 \mathrm{~g})$ with small $(\approx 0.02 \mathrm{~g})$ increments, and then 10 or $30 \mathrm{~cm}^{3}$ solution of SDS with known initial mass fraction, $w_{1, \mathrm{i}}$,was pipetted into each vessel. At this stage of the experiment the sorption systems were closed and their masses were measured again. In this manner all input parameters $\left(m_{\mathrm{t}}, m_{\mathrm{s}}\right.$ and $\left.w_{1, \mathrm{i}}\right)$ were exactly known. About twenty such closed systems were arranged in a plastic box which was immersed in a circulating bath at temperature $298 \pm 0.02 \mathrm{~K}$. Once or twice a day all bottles were gently shaken to promote attaining equilibrium. Since scattering of the data calculated from measurements were smaller for the systems $m_{\mathrm{t}}=30.0 \mathrm{~g}$, therefore majority of measurements were performed at this $m_{\mathrm{t}}$ value. At equilibrium first $1-2$ $\mathrm{cm}^{3}$ of supernatant solution was quickly pipetted into one the of $1 \mathrm{~cm}$ cells of the interferometer while the other, for comparison, was filled with distilled water. The cells were then closed and placed into the interferometer cell holder in order to ensure constant temperature, $298 \pm 0.02 \mathrm{~K}$, for the liquids. The interferometer (ITR-2, Russia) was calibrated with a series of SDS solutions in the range $0<w_{1}<0.03$ and the equilibrium mass fraction of SDS, $w_{1, \text { e }}$, was calculated from the equation fitted to the experimental points of the $w_{1}$ vs. readings curve. According to our experiences, the reproducibility of the readings was \pm 1.0 scale division so the overall accuracy 
of determination of SDS mass fraction was estimated to be $\pm 1-2 \times 10^{-5}$. All of the remaining part of the system was poured into a Petri dish and most of the equilibrium solution was removed by a special pipette, then the liquid film adhered to the surface of the swollen gel pieces were blotted with filter paper. After measuring the mass of the gel, it was dried until constant mass, so from the mass of swollen gel and $m_{\mathrm{s}}$, the mass degree of swelling, $q_{\mathrm{m}}$, and from the dried samples the mass of SDS inside the swollen gel could have been calculated. The mass ratio has been varied in the range $30<m_{\mathrm{t}} / m_{\mathrm{s}}<2500$. The sorption and swelling equilibrium measurements were performed at four initial mass fractions of SDS: 0.00576 (20 $\mathrm{mM}), 0.01152(40 \mathrm{mM}), 0.01728(60 \mathrm{mM})$ and $0.02304(80 \mathrm{mM})$. The code for the samples (networks) is: copolymer composition (VAc mole \%) / (RU/GDA).The code for the systems is: VAc, mole \% /(RU/GDA)/initial mass fraction of SDS. In majority of cases the measurements were repeated at least two times but for some selected systems (17.5/50/0.00576, $17.5 / 50 / 0.0115,17.5 / 50 / 0.0173,17.5 / 30 / 0.0115)$ the complete procedure was reproduced by independent persons (Z. S. and K. Sz.) including synthesis of gels as well.

\section{RESULTS AND DISCUSSION}

SMV Type of Isotherms and Related Quantities. When the SMV experimental and evaluation method is applied to a given adsorption/solvation system it is best to plot first the $m_{1}^{s v}=\mathrm{f}\left(w_{1, \mathrm{e}}\right)$ function in order to be able to check whether experimental data follow the linear

part of eq 3, or, as it cannot be excluded, both local (layer) composition and specific capacity vary in the entire range of the equilibrium concentration available for experiments. If the linearity, at least in a sensible range of the equilibrium concentration, is observed then both $k^{\mathrm{sv}}$ and $w_{1, l}$ can be calculated. Although it was taken for many other systems, in Figure 1 as 
examples, the $m_{1}^{s v} v s . w_{1, \mathrm{e}}$ functions are plotted at three initial mass fractions of SDS for the sample type $17.5 / 50$ and also for a system with higher degree of cross-linking $(17.5 / 30 / 0.01152)$. There is no doubt, at the lowest $w_{1, \mathrm{i}}(=0.00576)$ the upper part of the SMV isotherm separates definitely from the others and the linear parts of all fuctions have negative slope independently of the degree of cross-linking. These SMV isotherms bear out the typical way as surfactants bind to solid surfaces ${ }^{30}$ or as they used to interact with polymers. ${ }^{39,40}$

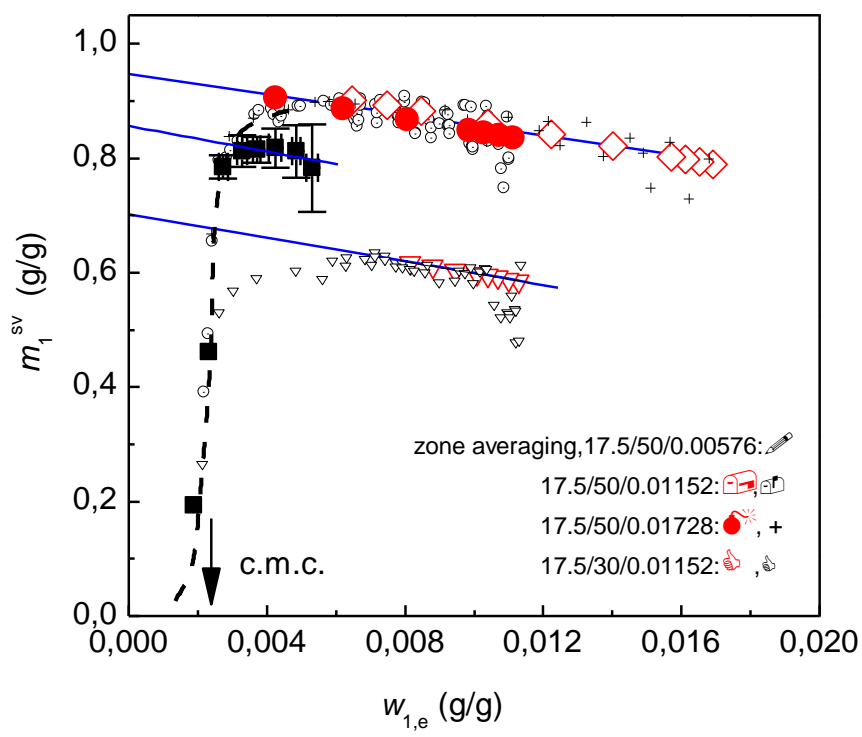

Figure 1. SMV isotherms for systems 17.5/50/0.00576, 17.5/50/0.01152, 17.5/50/0.01728 and 17.5/30/0.01152. The larger symbols were calculated from the smoothed $w_{1, \mathrm{e}}=\mathrm{f}\left(w_{\mathrm{s}}\right)$ curves. All other smaller symbols which belong to each curve denote $m_{1}{ }^{\text {sv }}$ values obtained from repeated and independent experiments; in this case data were substituted directly into eq 3 . Straight blue lines are linear fits to the smoothed data. For all of these systems the specific solvation capacity 
is $\approx 10 \mathrm{~g} / \mathrm{g}$. (For system $17.5 / 50 / 0.00576$ the systematic deviation from the linear approximation is much smaller than the experimental error.)

Although it was possible to estimate the slope and intercept from the 'crude' experimental data, much better results were obtained when first an appropriate part of $w_{1, \mathrm{e}} v s$. $w_{\mathrm{s}}$ functions had been fitted with polynomials or, slightly modified polynomials and then $m_{1}^{s v}$ values were calculated from these smoothed $w_{1, \mathrm{e}}$ data obtained from the fitted equation. These approximations are shown by solid lines in Figure 1. For some selected systems a detailed analysis of the experimental data has been performed in order to estimate experimental error. With the application of the so-called zone averaging method the necessary characteristics could have been calculated which are shown as error bars in Figure 1 (system:17.5/50/0.00576). (See, also Figure 1 and description, in Supporting Info. S3.). It is also shown by this figure that the interaction between surfactant and polymer gel commences below c.m.c. of SDS. With increasing SDS initial mass fraction the linear parts of the isotherms become longer and longer thus making possible more accurate determination of slope and intercept. Also, as a new finding, compared with S/L adsorption ${ }^{29}$, for systems 17.5/50/0.01152, 17.5/50/0.01728 and 17.5/50/0.02304 (this latter is not shown in Figure 1) the slope and intercept of the SMV isotherms were indistinguishable.

For all systems shown in Figure 1 the solvation specific capacity, $k^{\mathrm{sv}}$, was found to be surprisingly large $(\approx 10 \mathrm{~g} / \mathrm{g})$ compared it with that of the activated carbon of high specific surface area $(0.5-0.6 \mathrm{~g} / \mathrm{g}){ }^{29}$ Also, the average mass fraction of SDS in the solvation layer ( $w_{1, l}$ $=0.095)$ calculated from the slope and common intercept for systems $17.5 / 50 / 0.01152$ and $17.5 / 50 / 0.01728$ is more than ten times higher than its equilibrium values in the bulk phase (at 
$\left.w_{1, \mathrm{e}} \approx 0.006\right)$, i.e. the selectivity of this network for SDS is fairly high. In order to test further reliability and consistency of these data there is another possibility. Namely, to put the corresponding $k^{\mathrm{sv}}$ and $w_{1, l}$ values into a general equation derived earlier ${ }^{29}$

$$
w_{1, e}=\frac{\left(1-w_{s}\right) w_{1, i}-k^{s v} w_{1, l} w_{s}}{1-w_{s}\left(1+k^{s v}\right)}
$$

where meaning of the letters has already been given. The results of such calculations for network type 17.5/50 are shown in Figure 2: at lower values of $w_{\mathrm{s}}$ the agreement between measured and calculated functions is excellent. At greater $w_{\mathrm{s}}$ values the functions become nearly horizontal at $w_{1, \mathrm{e}}$ which correspond to the c.m.c. of SDS in the supernatant solution.

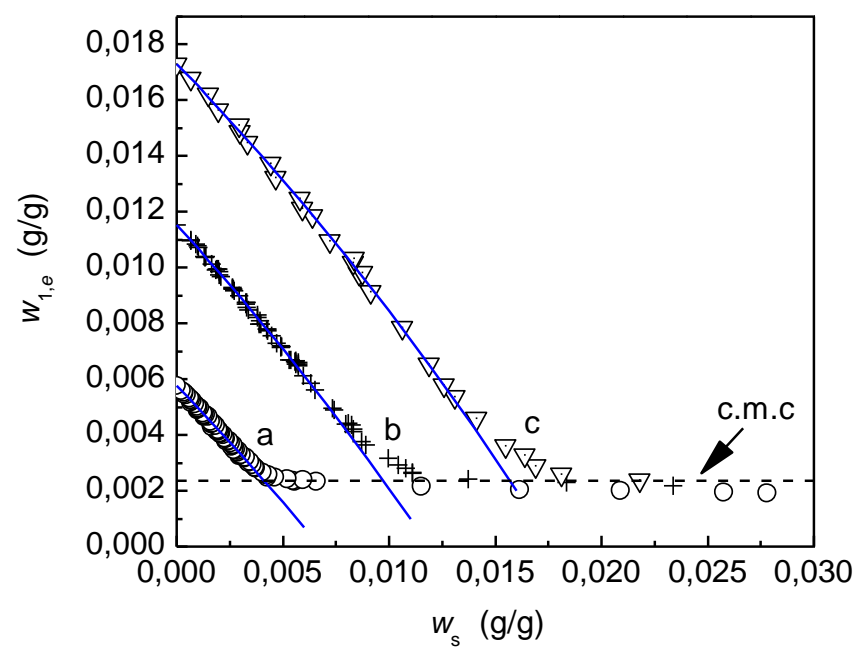

Figure 2. The effect of initial mass fraction of SDS on shape and position of the $w_{1, \mathrm{e}}=\mathrm{f}\left(w_{\mathrm{s}}\right)$ functions (sample 17.5/50). $a: w_{1, \mathrm{i}}=0.00576, b: w_{1, \mathrm{i}}=0.01152, c: w_{1, \mathrm{i}}=0.01780$. Solid blue curves have been calculated (eq 8) assuming constant $k^{\mathrm{sv}}$ and $w_{1, l}$ obtained from the slope and 
intercept of straight lines in Figure 1. $a: k^{\mathrm{sv}} \approx 10 \mathrm{~g} / \mathrm{g}, w_{1, l}=0.0870 ; b: k^{\mathrm{sv}}=10 \mathrm{~g} / \mathrm{g}, w_{1, l}=0.0948$; $c: k^{\mathrm{sv}}=10 \mathrm{~g} / \mathrm{g}, w_{1, l}=0.0954$.

In connection with eq 8 it is important to emphasize that it can be applied to describe any kind of $w_{\mathrm{e}}=\mathrm{f}\left(w_{\mathrm{s}}\right)$ functions if physically sensible data are substituted into it. If, however, a system reaches its limit $\left(w_{\mathrm{s}, \max }=1 /\left(1+k^{\mathrm{sv}}\right)\right)$, then $w_{1, \mathrm{i}}=w_{1, l}$, that means that supernatant mixture is not present any more in the adsorption/solvation system. If specific capacity is known, from rearrangement of eq 3, the local/layer average mass fraction of SDS can be calculated:

$$
w_{1, l}=\frac{m_{1}^{s v}}{k^{s v}}+w_{1, e}
$$

The results for network of type 17.5/50, at four initial mass fractions of SDS, are shown in Figure 3. As it can be expected, at $w_{1 . i}=0.00576$ the saturation value of $w_{1, l}$ is lower than that at higher values of $w_{1, \mathrm{i}}$ which, on the other hand, are practically independent of $w_{1, \mathrm{i}}$. This is again a new observation compared it with S/L adsorption system studied earlier. ${ }^{41}$

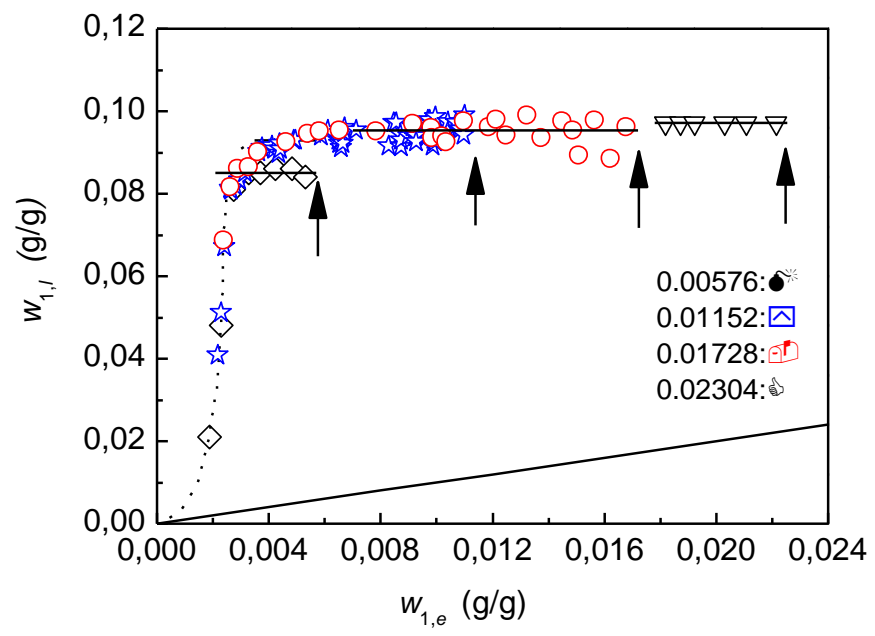


Figure 3. Dependence of the average mass fraction of SDS in the solvation layer on equilibrium mass fraction, $w_{1, \mathrm{e}}$ (type of network: 17.5/50). The arrows indicate initial mass fractions of SDS: 0.00576, 0.01152, 0.01728, and 0.02304. Horizontal solid lines: $w_{1, l}$ values were calculated from smoothed data. Straight line below shows 1:1 partitioning.

We have briefly discussed above the importance of the higher limit of $w_{\mathrm{s}}$ but, when the SMV method is applied, it is necessary to analyse its lower limit, too. This latter can be easily obtained if the linear parts of any of the isotherms shown in Figure 1 is extrapolated to their initial mass fractions, $w_{1, \mathrm{i}}$. For systems with common $w_{1, \mathrm{i}}$ no experimental point can exist beyond it. There is, however, another possibility as well. Namely, the partial differentiation of eq 8 with respect to $w_{\mathrm{s}}$ gives an equation which, when $w_{\mathrm{s}} \rightarrow 0$ (and so $w_{1, \mathrm{e}} \rightarrow w_{1, \mathrm{i}}$ ) reads

$$
m_{1}^{s v, 0}=-\left(\frac{\partial w_{1(e)}}{\partial w_{s}}\right)_{P, T, k^{s v}, w_{1, l}}=k^{s v}\left(w_{1, l}-w_{1, i}\right)
$$

where the differential quotient can be calculated from the initial slope of the $w_{1, \mathrm{e}}=\mathrm{f}\left(w_{\mathrm{s}}\right)$ function. The right-hand side of eq 10 is the specific solvation excess at this limit (differential or characteristic specific excess). Such points result in a function which does not depend on the ratio $m_{\mathrm{t}} / m_{\mathrm{s}}$ : it depends exclusively on the equilibrium mass fraction of the adsorbate (SDS). At the same time, it is the definition of the characteristic isotherm. Its knowledge is very important for multisorbent systems or, if the sorbent has different independent binding sites. Namely, by making use of the additivity rule, the isotherms for each individual sorbent component or site can in principle be calculated. ${ }^{41}$ It is not very well known that at finite values of $m_{\mathrm{s}}$ the usual excesses are not independent of each other. Therefore, in general, such a calculation cannot be correctly performed. It is interesting to note that in theoretical works on light scattering from multicomponent polymer solutions ${ }^{16,17}$ the differential quotient in eq 10 is used as a measure of 
preferential solvation. In Figure 4 such characteristic isotherm is shown for network type $17.5 / 50$ by making use of both methods for calculation of specific solvation excess at this limit. It is the first time when a characteristic isotherm is determined for binding of a surfactant. From the plot given in Figure 4 absolute specific value of the amount of SDS bound to the polymer chain can be given in other units, too. So, it is $3.34 \times 10^{-3} \mathrm{~mol} / \mathrm{g}$, or $0.171 \mathrm{~mol} \mathrm{SDS} / \mathrm{mol}$ repeat unit of copolymer. The reciprocal of the latter gives an approximate' stoichiometry' of the interaction: accordingly, nearly six repeat units (twelve $\mathrm{C}$ atoms in the main chain) bind one SDS molecule.

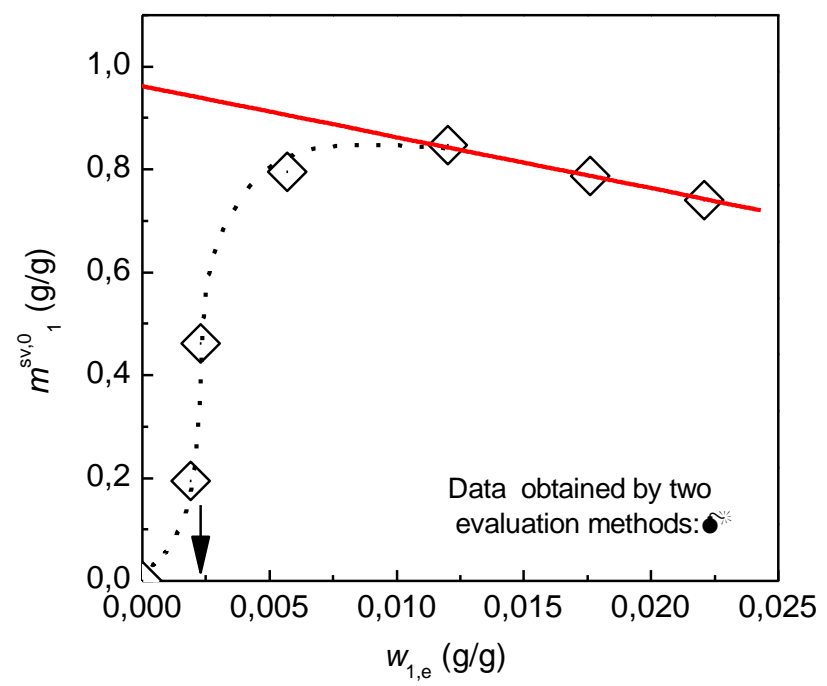

Figure 4. The characteristic isotherm for network type 17.5/50. The solid red line fits well to the last three SMV points making possible accurate determination of both slope and intercept $\left(k^{\mathrm{sv}}\right.$ and $\left.k^{\mathrm{sv}} \cdot w_{1, l}\right)$. The arrow shows location of c.m.c. $\left(k^{\mathrm{sv}}=9.92 \mathrm{~g} / \mathrm{g}, w_{1, l}=0.097\right.$ and $k^{\mathrm{sv}} \cdot w_{1, l}=$ $0.962 \mathrm{~g} / \mathrm{g}$ ) Dotted line: guide for the eyes in the non-linear region. 
In Figure 5 the comparision of a batch and two SMV isotherms illustrates again the possible difference between them; some agreement can be found only around the linear descending parts. However, the most interesting observation is the stepwise change in the specific solvation capacity for system when the value $w_{1, \mathrm{i}}$ is increased from 0.01152 to $0.02304 ; k^{\mathrm{sv}}$ drops from 22 $\mathrm{g} / \mathrm{g}$ to $11 \mathrm{~g} / \mathrm{g}$ while the specific amount bound remaines practically constant $\left(k^{\mathrm{sv}} \cdot w_{1, l}=1.12 \mathrm{~g} / \mathrm{g}\right)$. The very large specific solvation capacity cannot be an experimental error because similar values were obtained for other systems as well and, as will be discussed in another paragraph, it is consistent with a geometric model of solvation of polymer chains.

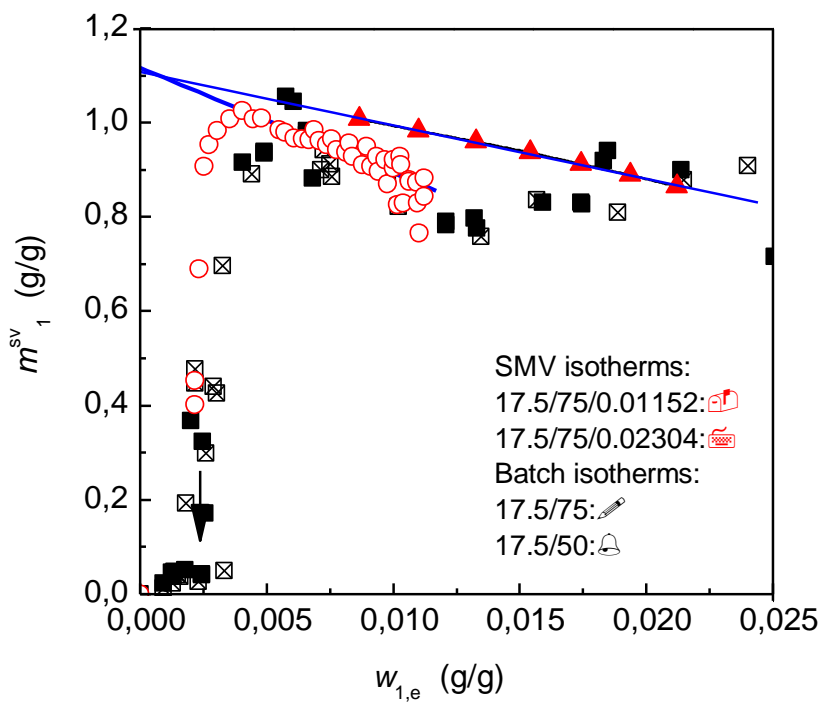

Figure 5. Comparision of SMV (17.5/75/0.01152 and 17.5/75/0.02304) and batch isotherms for networks: $17.5 / 75$ and $17.5 / 50$. The ratio $m_{\mathrm{t}} / m_{\mathrm{s}}=50\left(w_{\mathrm{s}} \approx 0.02\right.$ and $\left.m_{\mathrm{t}}=10 g\right)$ was kept constant during batch experiments. The arrow shows location of c.m.c. The linear approximations of the upper parts of SMV isotherms show a drastic change in specific solvation capacity. 
Dependence of Specific Solvaton Excess on Degree of Cross-linking. In the case of copolymer networks there are at least two essential, one might say, natural variables to deal with. One of these is the degree of cross-linking; the other is the composition and sequence of the copolymer which the networks are prepared from. As it has already been shown in Figure 1 the degree of cross-linking has a pronounced effect on binding ability of our gels: approximately 60 per cent increase in concentration of cross-linkages causes nearly 30 per cent decrease in the excess amount! According to our experiences, while $q_{\mathrm{m}}$ and $m_{1}^{s v, 0}$ was a quickly decreasing function of degree of cross-linking, the solvation specific capacity, especially for the looser gels, and at least in some range of degree of cross-linking, was nearly constant and smaller than the corresponding $q_{\mathrm{m}}$ (see, next paragraph, Figure 8). Although there are several important works discussing influence of the amount and internal structure of the cross-links or junction zones on properties of chemical and physical networks ${ }^{42,43,44}$, their role concerning preferential solvation is much less known. Nevertheless, it seems to be a reasonable assumption that the length of the network chains, at least to a lower limit, cannot affect solvation properties of polymer chains. However, in most cases the cross-linking reaction leads to a local chemical modification of the precursor polymer molecules and, as long as the amount of modified units is negligible, its effect on some properties is usually small. 


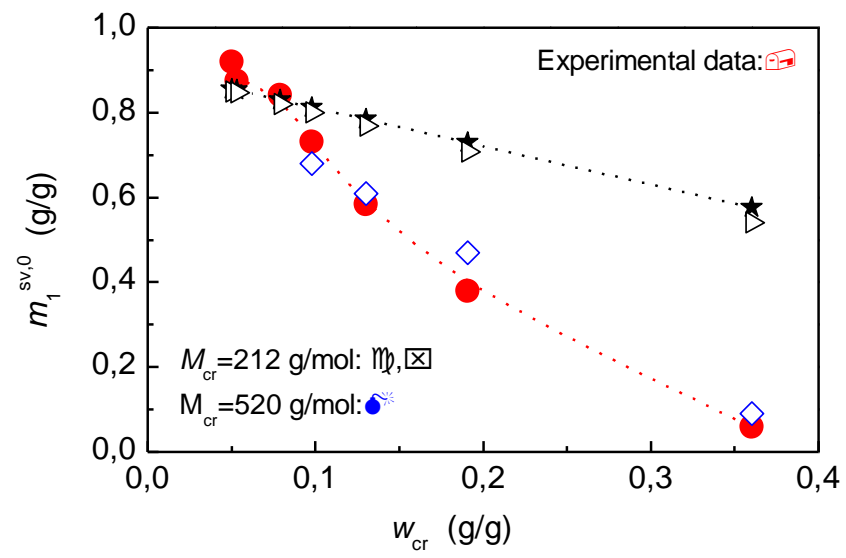

Figure 6. Experimentally determined dependence of the characterictic specific excess of preferential solvation on degree of cross-linking, $w_{\mathrm{cr}}$, for systems 17.5/x/0.01152: (x means each actual value of the independent variable; they are given here as $w_{\mathrm{cr}}$ ). If the smallest possible cross-link region is defined (molar mass of cross-link, $M_{\mathrm{cr}}$, is taken as $212 \mathrm{~g} / \mathrm{mol}$ ) to be part of the network where the specific excess is zero, or in the other case, total exclusion of SDS is assumed from the vicinity of cross-link (upper lines). For further detailes, see Text.

According to this view, it was assumed that the cross-link itself and its close molecular environment can be responsible for the effect observed. In our case four vinyl alcohol repeat units react with one mole GDA eliminating two moles of water so that in each chain 1,3 dioxane ring is formed which are then connected by a $-\left(\mathrm{CH}_{2}\right)_{3}-$ group. Thus, according to this local chemical structure we have defined the smallest cross-link as part of the whole network so that its molar mass, $M_{\mathrm{cr}}$, was taken $212 \mathrm{~g} \cdot \mathrm{mol}^{-1}$. From this and from the repeat units/GDA molar ratio, as well as from the average molar mass of the repeat unit, the mass fraction of cross-links, $w_{\mathrm{cr}}$, could have been calculated. In other words, $w_{\mathrm{cr}}$ measures relative contribution of the crosslinks to total mass of the dry network. In Figure 6 this is applied as independent variable to show variation of $m_{1}^{\mathrm{sv}, 0}$ as a function of degree of cross-linking. As can be seen, in the case of the highest degree of cross-linking (here molar ratio (RU/GDA) was 10:1) the gels have nearly lost their binding ability and the relative amount of cross-links in the network was more than 35 
mass per cent! All these mean that cross-links should definitely affect the preferential solvation of the copolymer chains in these systems. In order to estimate quantitatively the effect of crosslinks two 'mixed sorbent' models were chosen. In the first, zero excess was assumed for the

cross-links, so in eq $10 w_{1, l}=w_{1, \mathrm{i}}$ and the initial value, $m_{1, \text { in }}{ }^{\mathrm{sv}, 0}$, (when the effect of cross-link was considered to be negligible) was taken as $0.90 \mathrm{~g} / \mathrm{g}$. By making use of the additivity rule with $k^{\mathrm{sv}}=10 \mathrm{~g} / \mathrm{g}$ and at $w_{1, \mathrm{i}}=0.0114$ :

$$
m_{1, \text { mix }}^{s v, 0}=0.90\left(1-w_{c r}\right)+w_{c r} 10\left(w_{1, l}-w_{1, i}\right) \rightarrow m_{1, \text { mix }}^{s v, 0}=0.90\left(1-w_{c r}\right) .
$$

The other model assumes total exclusion of SDS from the vicinity of cross-links so in this case in eq $10 w_{1, l}=0$ and for the mixed sorbent

$$
m_{1, \text { mix }}^{s v, 0}=0,90\left(1-w_{c r}\right)+w_{c r} 10\left(0-w_{1, i}\right) \rightarrow m_{1, \text { mix }}^{s v, 0}=0,90\left(1-w_{c r}\right)+10 w_{c r}\left(-w_{1, i}\right) .
$$

The results are shown in Figure 6 as the two upper straight lines connecting the calculated points. Since the second term in eq 12 is small compared to the first one, the difference between the two models is small. At the same time, deviation from the experimental points is considerable except the range of low degree of cross-linking. However, if for $M_{\mathrm{cr}}$ a greater value, namely $520 \mathrm{~g} \cdot \mathrm{mol}^{-1}$ is chosen (cross-link plus $4-6$ repeat units) the agreement becomes much better and this shows also that the dependence of $M_{\mathrm{cr}}$ on degree of cross-linking is not a linear function. To illustrate the structural feature of the gel having the highest degree of crosslinking (molar ratio RU/GDA is 10:1) here the average number of repeat units between crosslinkes is about five, i.e. the network chains are shorter than full length of SDS molecule. So, for such a structural element or for such a network as a whole, a serious steric hindrance appears, particularly if one of the components of solution has relatively large molar volume.

The Effect of Copolymer Composition and Sequential Distribution. In Figure 7 dependence of compositional characteristics of the solvation layer on VAc content of 
copolymers are shown. In complete agreement with earlier investigations ${ }^{39}$ increasing hydrophobicity (VAc content) favors binding of SDS. Of the experimental results the points calculated for pure PVAc are remarkable. Since PVAc is insoluble in these dilute SDS solutions the points referring to it were calculated from data obtained for the 'blocky' type of copolymer and from data of pure PVA assuming additivity. These are shown at the end of curves denoted by $\mathrm{A}, \mathrm{B}$ and $\mathrm{C}$. If, however, $m_{1}^{s v, 0}$ values were calculated in the same way, but from data of the three statistical copolymers much higher values were obtained: as if the VAc blocks were less active in binding of SDS. It has been pointed out in our earlier work ${ }^{37}$ on swelling and mechanical properties of these copolymer gels that in the case of 'blocky' type of copolymers swollen in aqeous environment new junction zones were formed via association of the VAc blocks. That means that some parts of the polymer molecules are buried reducing both solvation specific capacity and binding ability of these sites.

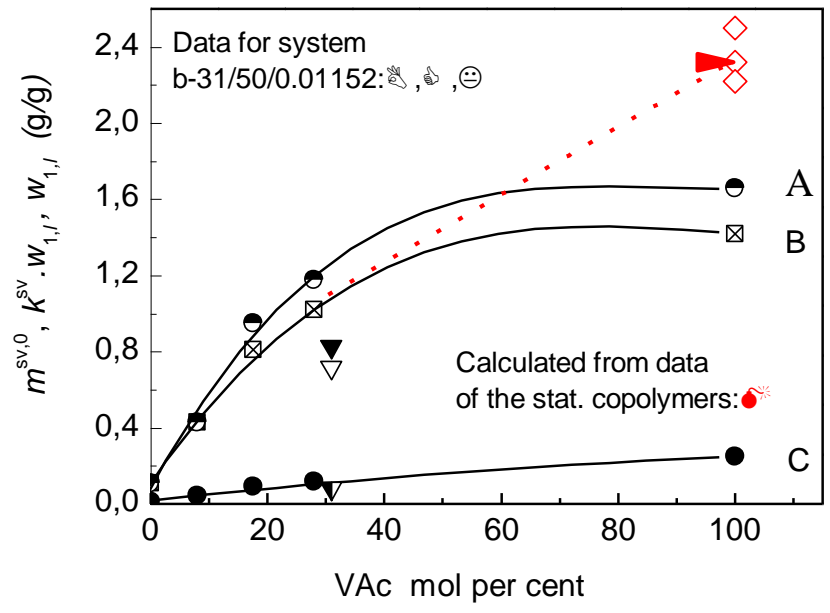


Figure 7. Dependence of compositional characteristics of solvation layer on VAc content of

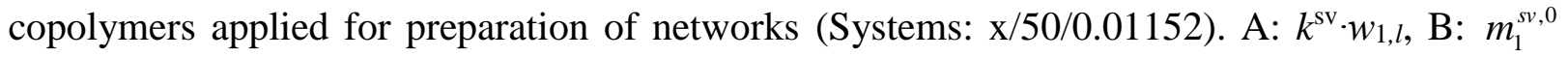
and $\mathrm{C}: w_{1, l}$ (x means the actual values of the independent variable). Solid lines are fitted polynomials of second and third order. The compositional characteristics of the solvation layer of the pure PVAc were calculated from these data and those referring to pure PVA assuming additivity.

Swelling and Solvation Specific Capacity. In terms of thermodynamics the swelling is a kind of phase equilibrium governed by equality of chemical potentials of the diffusible components, $\mathrm{i}$, in the outer and gel phase:

$$
\left(\Delta \mu_{i}\right)_{g e l}=\left(\Delta \mu_{i}\right)_{\text {out }} .
$$

Eq 13 refers to all diffusible components. Since the gel phase has minimum two components (network polymer + solvent), besides a mixing term which represents the driving force for swelling, the term $\left(\Delta \mu_{i}\right)_{g e l}$, as a consequence of swelling, should contain an additional contribution coming from deformation of the network. So, if no outer force or extra pressure is acting on a swollen network, the equilibrium condition can be given as

$$
\Delta \mu_{i, m i x}+\Delta \mu_{i, n e t}=\Delta \mu_{i, m i x}+P_{e l} \bar{V}_{i}=\left(\Delta \mu_{i}\right)_{\text {out }},
$$

where $P_{\mathrm{el}}$ is the so-called elastic potential, $\bar{V}_{i}$ is the partial molar volume of diffusible component $\mathrm{i}$, the meaning of $\Delta \mu_{i, n e t}$ is self-evident. In the case of neutral networks the mixing contribution is determined mainly by the network-solvent interaction while the elastic potential is a function of the degree of cross-linking. In general, at constant temperature and degree of cross-linking, $q_{\mathrm{m}}$ varies only a rather narrow range with variation of polymer-solvent interaction. In this respect the influence of the degree of cross-linking is more pronounced. On 
the other hand, larger change can be induced in degree of swelling by introducing even a small amount of electric charge into the network chains, i.e. in the case of polyelectrolyte gels.

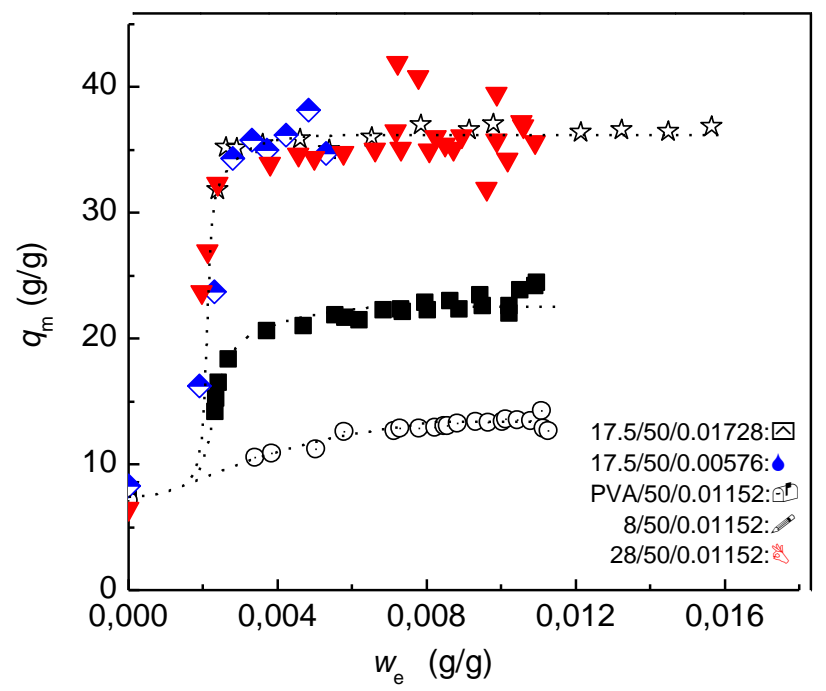

Figure 8. Dependence of mass degree of swelling, $q_{\mathrm{m}}$, on equilibrium mass fraction of SDS for networks at different $w_{1, \mathrm{i}}$ and with various copolymer compositions at constant degree of crosslinking.

As examples, in Figure 8 the dependence of $q_{m}$ on equilibrium mass fraction of SDS is shown for some selected systems. The similarity to and the close connection with the shape of the solvation isotherms ( $c f$. Figure 1) is obvious and it can be unambigously stated that the large change in degree of swelling is a consequence of transformation of neutral polymers into pseudo-polyelectrolytes through binding negatively charged SDS molecules to the network chains. The abrupt changes observed for some of the networks result in two-state type (switch on and off) systems which are very sensitive to the compositional variation of the environment. Similar phenomena were observed for other systems, too. ${ }^{45,46,47}$ If one were able to remove the 
bound SDS molecules from the solvation layer at molecular level by any special means, it would be possible to stimulate such kind of thin gel fiber resulting in a quick change, let us say, in its length. One possibility to do so is to apply competitive interaction by adding a new component to such a system. It has been shown by some preliminary experiments that addition of some amount of $\beta$-cyclodextrin or 1-propanol at the swelling maximum to a PVAc-SDS gel caused sharp decrease in degree of swelling which, on the other hand, indicates the complete reversibility of binding.

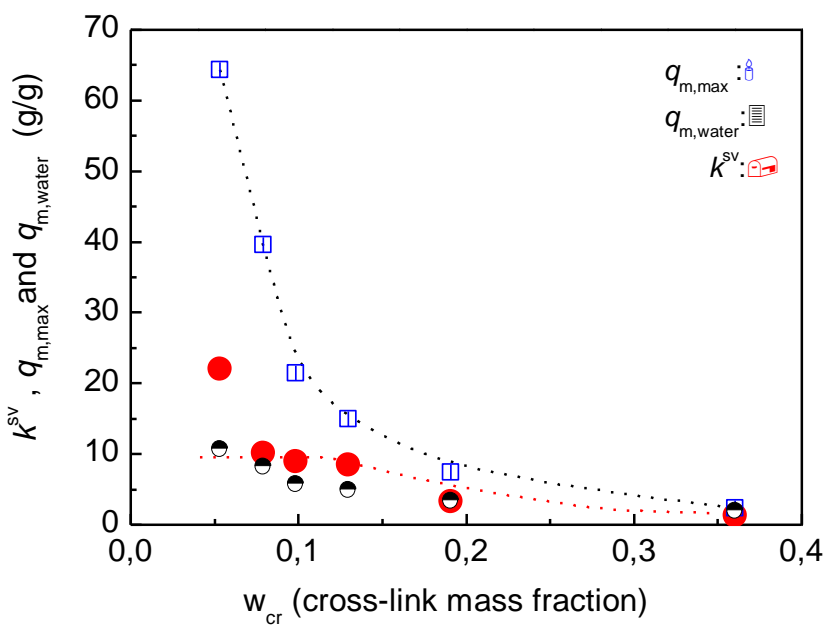

Figure 9. Dependence of the maximum degree of swelling, $q_{\mathrm{m}, \max }$, in SDS solution, the degree of swelling in water, $q_{\mathrm{m} \text {,water }}$ and solvation specific capacity, $k^{\mathrm{sv}}$, on degree of cross-linking. Systems: $17.5 / \mathrm{x} / 0.01152$ (x means each actual value of the independent variable now given as $\left.w_{\mathrm{cr}}\right)$.

In Figure 9 the dependence of degree of swelling and solvation specific capacity are given as a function of degree of cross-linking. According to eq 5, $q_{\mathrm{m}, \max }$ should always be greater than even the largest capacity value and it is a quickly decreasing function of the degree of cross- 
linking. At the same time, solvation specific capacity, $k^{\mathrm{sv}}$, disregarding from the initial jumplike change, is nearly constant then it begins to decrease slowly parallel with $q_{\mathrm{m} \text {,max }}$.

Simple Geometric Model of the Solvation Layer. In order to find explanation for the high specific solvation capacity values a cylindrical core/shell geometric model was put forward: if the diameter of the core is $d_{\text {core }}$ and the outer diameter of the shell is $d_{\text {shell }}$ then, the maximum available 'volumetric' specific capacity can be calculated if the $d_{\text {shell }} / d_{\text {core }}$ is assumed to be three (monolayer formed around the core):

$$
\begin{aligned}
& k_{\max }^{s v}=\left[(\text { volume })_{\text {core }+ \text { shell }}-(\text { volume })_{\text {core }}\right] /(\text { volume })_{\text {core }}, \text { or } \\
& k_{\max \text { mono }}^{s v}=\left[\left(3 d_{\text {core }} / 2\right)^{2}-\left(d_{\text {core }} / 2\right)^{2}\right] /\left(d_{\text {core }} / 2\right)^{2}
\end{aligned}
$$

In this case the simplest model gives $k_{\max , \text { mono }}^{s v}=8.0$ for the specific capacity. This value is relatively close to the one calculated from the experimental data for systems represented in Figure 1. Of course, in the case of these models the size of the core, thickness of the shell or their ratio can be varied in a wide range in order to fit calculated data to the experimental finding. So, if the diameter of core is increased by 20 per cent, then $k_{\max \text {,mono }}^{s v}$ will decrease to 6.11; if, however the thickness of the shell is increased by 20 per cent $k_{\max \text { mono }}^{\text {sv }}$ goes up to 10.56 which is close to specific capacities obtained for network type 17.5/50 at different initial SDS mass fractions ( $c f$. caption of Figure 2 ). For a bilayer, where $d_{\text {shell }}=5 d_{\text {core, }}$ a sudden change, namely $k_{\max b i}^{s v}=24.0$ can be estimated which is close to the values $k^{\mathrm{sv}}=22$ and $k^{\mathrm{sv}}=21.3$ obtained from experiments for systems 17.5/75/0.01152 and 28/50/0.01152, respectively. Although it is continuum and static by nature, the flexibility and versatility of the geometric model proved to be very useful. In real systems, however, long chain molecules and the carbon 
chains of SDS molecules cannot be considered simply as straight rods because fluctuation results in changes continuously in their local conformation. Also, from our experimental data it cannot be estimated how much can be the contribution of a (highly compressed) electric double layer to the specific capacity, or the difference in cross-section between the alkyl chain and hydrated polar head of SDS molecules. What we can state for certainty it is the most probable position of SDS molecules: they should be oriented nearly parallel with the axis of the polymer chain being either in single or aggregated form. The higher value of specific capacity $(\approx 20 \mathrm{~g} / \mathrm{g})$ substantiates the presence of larger aggregates (aggregation number around 20-40) surrounding the polymer chains. (Further detailes are summarized in Table 3. and Figure 2., Supporting Info. S4 and S5.)

\section{CONCLUSION}

According to a classification of $\mathrm{S} / \mathrm{L}$ isotherms by Kipling-Schay-Nagy ${ }^{6,28}$ our SMV isotherms correspond to type III or type IV with differences that, instead of a continuously increasing ascending part, the binding isotherm of SDS is of sigmoidal shape and shifted to the very low $\left(10^{-4}\right)$ mole fraction range. This finding is very important from another point of view as well; in this case the SMV and batch isotherms partly coincide so they mutually support the accepted view on constancy of layer composition in the linear region. ${ }^{35}$ If the range of composition available is strongly limited, for instance by the limited solubility of the adsorbate, then the SMV method is superior to any others: it requires only one initial concentration to determine local characteristics of an adsorption/solvation system.

On the basis of the present and our earlier works ${ }^{29,41}$ it is assumed that deviation between the SMV and batch isotherms is a general phenomenon and the coincidence of the two kinds of 
approximation is an exception. The shape and position of the $w_{1, l} v s . w_{1, \mathrm{e}}$ functions is the key factor to understand the situation. If they have a horizontal (constant) part as $w_{1, \mathrm{e}} \rightarrow w_{1, \mathrm{i}}$ and value of $w_{1, l}$ depends also on $w_{1, \mathrm{i}}$, separation should result. If, however, $w_{1, l}$ is constant (i.e. independent of $w_{1, \mathrm{e}}$ and $\left.w_{1, \mathrm{i}}\right)$ in a wide range of mass fraction, $w_{1, \mathrm{e}}$, then, both points belonging to a batch isotherm and SMV points, including the characteristic points of the SMV isotherm, fall on the same linear function.

The structure of networks affects not only the local average composition but the structure of the solvation layer, too. Namely, it is assumed that to gels of the two highest degree of crosslinking, the SDS molecules bind one by one in a 'singlet' form; at moderate degree of crosslinking mainly in the form of monolayered cylindrical aggregates (aggregation number 6-20) and for loosely cross-linked networks or for those having high binding ability in the form of bilayered cylindrical aggregates (aggregation number 20-40). All these are supported by the experimental facts that with increasing degree of cross-linking the 'cooperativity' (steepness) of the $m_{1}^{s v}=\mathrm{f}\left(w_{1, \mathrm{e}}\right)$ and $q_{\mathrm{m}}=\mathrm{f}\left(w_{1, \mathrm{e}}\right)$ functions gradually decreases. It is important to emphasize here: if the binding happens in the form of aggregates, average value of $w_{1, l}$ may include extremities.

The SMV method opens up several new possibilities for further research: in order to ensure its widest generality it would be important to test its applicability for classical systems by independent laboratories. Theoretical works are needed to prove unambigouosly the shape of the $w_{1, l}=\mathrm{f}\left(w_{1, \mathrm{e}}\right)$ functions found experimentally in the present and earlier ${ }^{29,41}$ works. It would be very interesting to use this consideration when the so-called in situ methods are applied for determination of local composition. A very essential problem: how the SMV method can be 
applied to different types of interfaces. The experimental facts in this and our earlier works ${ }^{29,41}$ have convinced us that the concept of SMV isotherm is a powerful one and may contribute to understanding better solvation/adsorption phenomena. A succesful generalization would mean that some of the accepted rules and definitions concerning preferential solvation and adsorption need to be supplemented and modified.

\section{AUTHOR INFORMATION}

\section{Corresponding Author}

*E-mail: miklosnagy@chem.elte.hu

\section{Notes}

The authors declare no competing financial interest

\section{ACKNOWLEDGEMENTS}

The authors are grateful to all members of Laboratory for Colloid and Supermolecular Structures for their permanent support and for the financial help by National Science Foundation (OTKA, T22935) for elaboration of synthesis of polymers and networks. The authors thank to Mrs. Jaksity for her invaluable help during experimental works.

\section{REFERENCES}

(1) Langmuir, I. The Adsorption of Gases on Plane Surfaces of Glass, Mica and Platinum. $J$. Am. Chem. Soc. 1918, 40, 1361-1403.

(2) Hückel, E. Adsorption und Kapillarkondenzation; Leipzig, 1928. 
(3) Brunauer, S.; Emmett, P. H.; Teller, E. Adsorption of Gases in Multimolecular Layers. J. Am. Chem. Soc. 1938, 60, 309-319.

(4) Brunauer, S.; Deming, L. S.; Deming, W. E.; Teller, E. On a Theory of the van der Waals Adsorption of Gases. J. Am. Chem. Soc. 1940, 62, 1723-1732.

(5) Brunauer, S. The Adsorption of Gases and Vapors; Princeton, 1945.

(6) Kipling, J. J. Adsorption from Solutions of Non-Electrolytes; Academic Press, London, New York, 1965.

(7) Schay, G. Adsorption of Solution of Nonelectrolytes; Surface and Colloid Science, Vol. 2. Ed.: Matijevič, E.; Wiley, London, 1969.

(8) Parfitt, G. D.; Rochester, C. H. Adsorption from Solutions at the Solid/Liquid Interface; Academic Press, New York, London, 1983.

(9) Scatchard, G. The Sorting of Mixed Solvents by Ions. J. Chem. Phys. 1941, 9, 34-41. (10) Grunwald, E.; Baughman, G.; Kohnstam, G. The Solvation of Electrolytes in DioxaneWater Mixtures as Deduced from the Effect of Solvent Change on the Standard Partial Molar Free Energy. J. Am. Chem. Soc. 1960, 82(22), 5801-5811.

(11) Padova, J. Ion-solvent interaction. VI. Thermodynamic approach to preferential solvation in mixed solvents. J. Phys. Chem. 1968, 72, 796-800.

(12) Ichikawa, T.; Kevan, L. Solvation-shell geometry of copper ion in methanol and in acetone and a molecular picture of preferential solvation of copper ion by methanol in methanol-acetone mixed solvent determined by electron spin-echo modulation analysis. $J$. Phys. Chem. 1980, 84 (15), 1955-1958. 
(13) Kobara, H.; Wakisak, A.; Takeuchi, K.; Ibusuki, T. Preferential Solvation of $\mathrm{Na}^{+}$in $N, N^{\prime}$

- Dimethylformamide - Water Binary Mixture. J. Phys. Chem. B, 2003, 107 (43),

11827-11829.

(14) Banerjee, D.; Laha, A. K.; Bagchi, S. Preferential solvation in mixed binary solvent. J. Chem. Soc. Faraday Trans. 1995, 91, 631-636.

(15) Amenta, V.; Cook, J. L.; Hunter, Ch. A.; Low, C. M. R.; Vinter, J. G. Influence of Solvent Polarity on Preferential Solvation of Molecular Recognition Probes in Solvent Mixtures. J. Phys. Chem. B, 2012, 116 (49), 14433-14440.

(16) Ewart, R. H.; Roe, C. P.; Debye, P.; McCartney, J. R. The Determination of Polymeric Molecular Weights by Light Scattering in Solvent-Precipitant Systems. J. Chem. Phys. 1946, $14,687-695$.

(17) Stockmayer, W. H. Light Scattering in Multi-Component Systems. J. Chem. Phys. 1950, $18,58-61$.

(18) Flory, P. J. Principles of Polymer Chemistry 1st ed.; Cornell University Press: Ithaca, N.Y., 1953; Chapter XIII.

(19) Strazielle, C.; Benoit, H. Theoretical study of the scattering of light by ternary systems. J. Chim. Phys. Phys.- Chim. Biol. 1961, 58, 675-677.

(20) Read, B. E. A light-scattering study of preferential adsorption in the system benzene + cyclohexane + polystyrene. Trans. Faraday Soc. 1960, 56, 382-390. 
(21) Davis-Searles, P. R.; Saunders, A. J.; Erie, D. A.; Winzor, D. J.; Pielak, G. J. Interpreting the Effects of Small Uncharged Solutes on Protein - folding Equilibria. Annu. Rev. Biophys. Biomol. Struct. 2001, 30, 271-306.

(22) Casassa, E. F.; Eisenberg, H. On the Definition of Components in Solutions Containing Charged Macromolecular Species. J. Chem. Phys. 1960, 64, 753-756.

(23) Okita, K.; Teramoto, A.; Kawahara, K.; Fujita, H. Light scattering and refractometry of a monodisperse polymer in binary mixed solvents. J. Phys. Chem. 1968, 72, 278-285.

(24) Chatterjee, P.; Bagchi, S. Study of preferential solvation in mixed binary solvents by ultraviolet-visible spectroscopy. J. Chem. Soc. Faraday Trans. 1990, 86, 1785-1789.

(25) Laha, A. K.; Das, P. K.; Bagchi, S. Study of Preferential Solvation in Mixed Binary Solvent as a Function of Solvent Composition and Temperature by UV-Vis Spectroscopic Method. J. Phys. Chem. A. 2002, 106 (13), 3230-3234.

(26) Frankel, L. S.; Langford, C. H.; Strengl, T. R. Nuclear magnetic resonance techniques for the study of preferential solvation and the thermodynamics of preferential solvation. .J. Phys. Chem. 1970, (6), 1376-1381.

(27) Ben-Naim, A. Preferential solvation in two- and in three-component systems. Pure \& Appl. Chem. 1990, Vol. 62, No. 1, 25-34.

(28) Schay, G.; Nagy, L. G. Elegyadszorpció folyadék/szilárd és folyadék/gőz határfelületeken; (A kémia újabb eredményei, 18. ed. Csákvári Béla) Akadémiai Kiadó, Budapest, 1974. 
(29) Nagy, M. Experimental study of adsorption from dilute aqueous solutions of a nonelectrolyte: 1-propanol on activated carbon. Langmuir 1991, 7, 344-349.

(30) Zhang, R.; Somasunduran, P. Advances in adsorption of surfactants and their mixture at solid/solution interfaces. Adv. Colloid and Interface Sci., 2006, 123, 213-229.

(31) Everett, D. H. Thermodynamics of adsorption from non-aqueous solutions. Progr. Colloid \& Polymer Sci. 1978, 65, 103-117.

(32) Klinkenberg, A. Isotherms for preferential adsorption from binary mixtures based on Langmuir equation. Rec. Trav. Chim. 1959, 78, 83-90.

(33) Conford, P. V.; Kipling, J. J.; Wright, E. H. T. Adsorption from solution: thickness of the adsorbed layer. Trans. Faraday Soc. 1962, 58, 74-85.

(34) Everett, D. H. Thermodynamics of adsorption from solution. Part 1. - Perfect systems. Trans. Faraday Soc. 1964, 60, 1803-1813.

(35) Nagy, L. Gy. Folyadékelegyek adszorpciója. Kémiai Közlemények 1967, 27/3, 323-359.

(36) Tubbs, R. K. Sequence Distribution of Partially Hydrolyzed Poly(vinyl Acetate). J. Polymer Sci. A-1 1966, 4, 623-629.

(37) Nagy, M.; Györgyiné Edelényi, J. Association - aggregation phenomena in polymer gels, I., Effect of the condition of gel preparation on the swelling and mechanical properties of poly(vinyl alcohol, vinyl acetate) copolymer gels. Acta Chim. Acad. Sci. 1989, 126/4, 507-517.

(38) Nagy, M. A comparative and systematic thermodynamic study of aqueous solutions and hydrogels of homo- and copolymers of poly(vinyl alcohol). Physical Chemistry Chemical Physics 2000, 2, 2613-2622. 
(39) Gilányi, T.; Wolfram, E. Complex formation between ionic surfactants and polymers in aqueous solution, in: P. Dubin (ed.) Microdomains in Polymer Solutions; Plenum Press, New York, 1985.

(40) Tam, K. C.; Wyn-Jones, E. Insights on polymer surfactant complex structures during the binding of surfactants to polymers as measured by equilibrium and structural techniques. Chem. Soc. Rev. 2006, 35, 693-709.

(41) Nagy, M. Study of adsorption from dilute solutions: Thermodynamic analysis of experimental data. Langmuir 1994, 10, 563-569.

(42) Dušek, K.; Prins, W. Structure and Elasticity of Non-Crystalline Polymer Networks. Adv. Polymer Sci. 1969, Vol.6, 1-102.

(43) Clark, A. H.; Ross-Murphy, S. B. Structural and Mechanical Properties of Biopolymer Gels. Adv. Polymer Sci. 1987, 83, 57-192.

(44) Keller, A. Introductory lecture. Aspects of polymer gel. Faraday Discuss. 1995, 101, $1-49$.

(45) Rosén, O.; Piculell, L.; Hourdet, D. Swelling of Poly(acrylamide) Gels with Pendant Poly(ethylene oxide) Chains in Solution of Ionic Surfactant and Salt. Langmuir 1998, 14 $777-782$.

(46) Sjöström, J.; Piculell, L. Simple Gel Swelling Experiments Distinguish between Associating and Nonassociating Polymer - Surfactant Pairs. Langmuir 2001, 17, 3836-3843. 
(47) Lynch, I.; Sjöström, J.; Piculell, L. Hydrophobicity and Counterion Effect on the Binding of Ionic Surfactants to Uncharged Polymeric Hydrogels. J. Phys. Chem. B 2005, 109, $4252-4257$.

Supporting Information Available: Detailes of condition of formation and basic characteristics of precursor polymers and networks; An example to show some detailes of the zone averaging method; Solvation and swelling data for copolymer gels with various composition and degree of cross-linking; Comparision of the SMV isotherms of statistical and 'blocky' type of copolymer networks.

This material is available free of charge via Internet at http://pubs.acs.org. 
The Sorbent Mass Variation Method: a New Possibility for Determination of Binding

Isotherms

Miklós Nagy*, Zoltán Siegl, Krisztina Szili, Viktória Fábos and Krisztina Kántor

Table of content (TOC):

Sorbent Mass Variation (SMV) method

Experimental input function
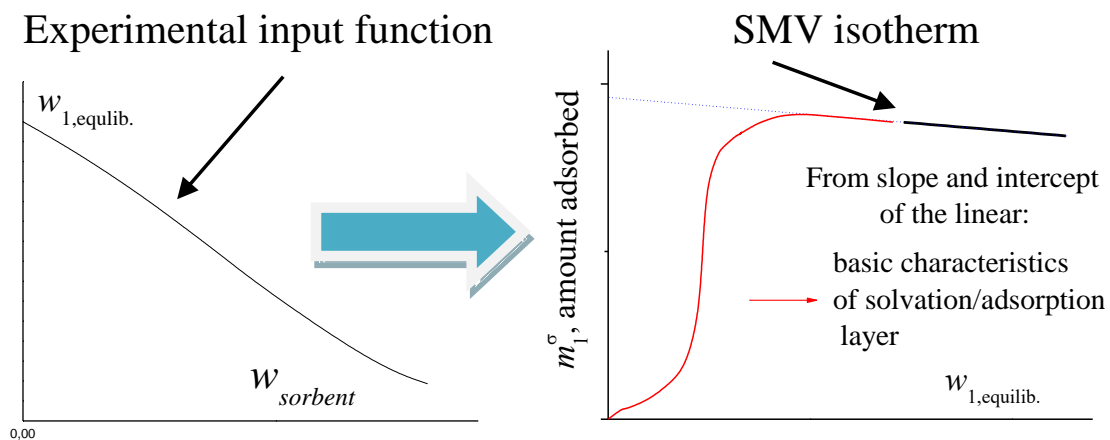

$$
\text { of the linear: }
$$$$
\text { of solvation/adsorption }
$$$$
\text { 1,equilib }
$$ 